\title{
Some Invariants of Cartesian Product of a Path and a Complete Bipartite Graph
}

\author{
Ramy Shaheen, Suhail Mahfud, Qays Alhawat \\ Department of Mathematics, Faculty of Science Tishreen University, Lattakia, Syria \\ Email address: \\ shaheenramy2010@hotmail.com (R. Shaheen), shaheenramy@tishreen.edu.sy (R. Shaheen), suhailmahfd@yahoo.com (S. Mahfud), \\ kais.hw007@gmail.com (Q. Alhawat)
}

\section{To cite this article:}

Ramy Shaheen, Suhail Mahfud, Qays Alhawat. Some Invariants of Cartesian Product of a Path and a Complete Bipartite Graph. International Journal of Discrete Mathematics. Vol. 4, No. 2, 2019, pp. 61-70. doi: 10.11648/j.dmath.20190402.11

Received: November 11, 2019; Accepted: Decmeber 16, 2019; Published: January 6, 2020

\begin{abstract}
A topological index of graph $\mathrm{G}$ is a numerical parameter related to $\mathrm{G}$, which characterizes its topology and is preserved under isomorphism of graphs. Properties of the chemical compounds and topological indices are correlated. In this paper we will compute M-polynomial, first and second Zagreb polynomials and forgotten polynomial for the Cartesian Product of a path and a complete bipartite graph for all values of $\mathrm{n}$ and $\mathrm{m}$. From the M-polynomial, we will compute many degreebased topological indices such that general Randić index, inverse Randić index, first and second Zagreb index, modified Zagreb index, Symmetric division index, Inverse sum index augmented Zagreb index and harmonic index for the Cartesian Product of a path and a complete bipartite graph. Also, we will compute the hyper- Zagreb index, the first and second multiple Zagreb index and forgotten index for the Cartesian Product of a path and a complete bipartite graph.
\end{abstract}

Keywords: M-polynomial, Topological Index, Path, Complete Graph, Cartesian Product

\section{Introduction}

Through this paper we consider simple connected graph, i.e. connected without loops and multiple edges. Let $G(V, E)$ be a graph with vertex and edge are sets of $V(G)$ and $E(G)$, respectively. The degree of vertex $u$ in $G$ is denoted by $d_{u}$, which is defined as the number of edges incident to $u$.

In chemical graph theory, a molecular graph is a simple graph (having no loops and multiple edges) in which atoms and chemical bonds between them are represented by vertices and edges respectively. A graph $G(V, E)$ with vertex set $V(G)$ and edge set $E(G)$ is connected if there exists a connection between any pair of vertices in $G$.

A topological index is a function that characterizes the topology of the graph. Most commonly known invariants of such kinds are degree-based topological indices. These are actually the numerical values that correlate the structure with various physical properties, chemical reactivity and biological activities.

Numerous graph polynomials were introduced in the literature, several of them turned out to be applicable in mathematical chemistry. There are relationships between topological indices and polynomials, and we can know a lot of indices, when we know polynomials for instance, the Hosoya polynomial [1], see also [2-4], is the key polynomial in the area of distance-based topological indices. In particular, the Wiener index which is the first topological index introduced by chemist Harold Wiener [5, 6] can be computed as the first derivative of the Hosoya polynomial, evaluated at 1, the hyper-Wiener index [7] and the TratchStankevich-Zefirov index can be obtained similarly [8].

M-polynomial [9], introduced by Deutsch and Klavzar in 2015 , plays the same role in determining closed forms of many degree-based topological indices $[10,11]$. The main advantage of M-polynomial is the wealth of information that it contains about degree-based graph invariants and by finding M-polynomial we can conclude many degree-based topological indices as we will find in the definitions of the article.

The Cartesian product $G H$ of two graphs $G$ and $H$ is the graph with vertex set $V(G H)=V(G) \times V(H)$, where two vertices $\left(v_{1}, v_{2}\right),\left(u_{1}, u_{2}\right) \in V(G H)$ are adjacent if and only if either $v_{1} u_{1} \in E(G)$ and $v_{2}=u_{2}$ or $v_{2} u_{2} \in E(H)$ and $v_{1}=u_{1}$.

A path graph $P_{n}$ is a graph whose vertices can be listed in the order $v_{1}, v_{2}, \ldots, v_{n}$ such that the edges are $\left\{v_{i}, v_{i+1}\right\}$, where $i=1,2, \ldots, n-1$. A path $P_{n}$ consists of two vertices 
of degree one and $n-2$ vertices of degree two.

complete bipartite graph $K_{(n, m)}$ is a graph whose vertices can be partitioned into subsets $V_{1}, V_{2}$ such that no edge has both endpoints in the same subset, and every possible edge that could connect vertices in different is part of the graph if $n=1$ then the complete bipartite graph is called star graph.

Figure 1 shows a Cartesian Product of path and complete bipartite graph $P_{3} K_{(1,4)}$.

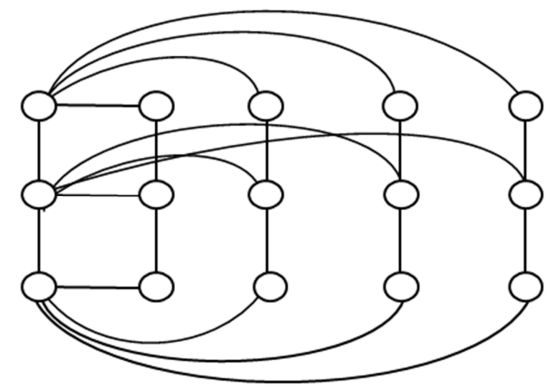

Figure 1. $P_{3} K_{(1,4)}$.

\section{Basic Definitions and Literature Review}

Here we give some basic definitions and literature review. Definition 2.1. The M-polynomial of $G$ is defined in [9] as:

$$
M(G, x, y)=\sum_{\delta \leq i \leq j \leq \Delta} m_{i j} x^{i} y^{j}
$$

where $\delta=\min \left\{d_{v}: v \in V(G)\right\}, \Delta=\max \left\{d_{v}: v \in V(G)\right\}$, and $m_{i j}(G)$ is the number of edges $e=v u \in \mathrm{E}(\mathrm{G})$ such that $d_{v}=i, d_{u}=j$.

Gutman and Trinajstic [12] introduced first Zagreb index and second Zagreb index,

$$
\begin{gathered}
M_{1}(G)=\sum_{u v \in \mathrm{E}(\mathrm{G})}\left(d_{u}+d_{v}\right) \text { and } \\
M_{2}(G)=\sum_{u v \in \mathrm{E}(\mathrm{G})}\left(d_{u} \times d_{v}\right)
\end{gathered}
$$

respectively. For details about these indices we refer [13-17] to the readers. Both the first Zagreb index and the second Zagreb index give greater weights to the inner vertices and edges, and smaller weights to the outer vertices and edges which oppose intuitive reasoning [18]. For a simple connected graph $\mathrm{G}$, the second modified Zagreb index is defined as:

$$
{ }^{\mathrm{m}} M_{2}(G)=\sum_{u v \in E(G)} \frac{1}{d_{u} \times d_{v}} .
$$

Randić index, [19] denoted by $R_{-1 / 2}(G)$ and introduced by Milan Randić in 1975, is also one of the oldest topological indices. The Randić index is defined as

$$
R_{-\frac{1}{2}}(\mathrm{G})=\sum_{u v \in \mathrm{E}(\mathrm{G})} \frac{1}{\sqrt{d_{u} d_{v}}}
$$

In 1998, working independently, Bollobas and Erdos [20] and Amic et al. [21] proposed the generalized Randić index and it has been studied extensively by both chemists and mathematicians [22].

The general Randić index is defined as: $R_{\alpha}(G)=$ $\sum_{u v \in \mathrm{E}(\mathrm{G})} \frac{1}{\left(d_{u} d_{v}\right)^{\alpha}}$ and the inverse Randić index is defined as: $R R_{\alpha}(G)=\sum_{u v \in \mathrm{E}(\mathrm{G})}\left(d_{u} d_{v}\right)^{\alpha}$. Obviously, $R_{-1 / 2}(\mathrm{G})$ is the particular case of $R_{\alpha}(\mathrm{G})$ when $\alpha=\frac{1}{2}$.

The augmented Zagreb index of $G$ proposed by Furtula et al. [23] is defined as:

$$
A(\mathrm{G})=\sum_{u v \in \mathrm{E}(\mathrm{G})}\left\{\frac{d_{u} d_{v}}{d_{u}+d_{v}-2}\right\}^{3}
$$

and it is useful for computing heat of formation of alkanes $[23,24]$.

The symmetric division index [SDD] is the one among 148 discrete Adriatic indices and is a good predictor of the total surface area for polychlorobiphenyls, see [25]. The symmetric division index of a connected graph $\mathrm{G}$ is defined as:

$$
S D D(G)=\sum_{u v \in \mathrm{E}(\mathrm{G})}\left\{\frac{\min \left(d_{u}, d_{v}\right)}{\max \left(d_{u}, d_{v}\right)}+\frac{\max \left(d_{u}, d_{v}\right)}{\min \left(d_{u}, d_{v}\right)}\right\} .
$$

The inverse sum index is the descriptor that was selected in [26] as a significant predictor of total surface area of octane isomers and for which the extremal graphs obtained with the help of mathematical chemistry have, particularly, a simple and elegant structure. The inverse sum index is defined as:

$$
I(G)=\sum_{u v \in \mathrm{E}(\mathrm{G})} \frac{d_{u} d_{v}}{d_{u}+d_{v}}
$$

Another variant of Randić index is the harmonic index defined as:

$$
H(\mathrm{G})=\sum_{u v \in \mathrm{E}(\mathrm{G})} \frac{2}{d_{u}+d_{v}}
$$

As far as we know, this index first appeared in [27]. Favaron et al. [28] considered the relation between the harmonic index and the eigenvalues of graphs.

We give derivations of some well-known degree-based topological indices from M-polynomial [9] in Table 1.

Table 1. Derivations of some degree-based topological indices from $M$ polynomial.

\begin{tabular}{ll}
\hline Topological Index & Derivation from $\boldsymbol{M}(\boldsymbol{G} ; \boldsymbol{x}, \boldsymbol{y})$ \\
\hline First Zagreb & $\left(D_{x}+D_{y}\right)(M(G ; x, y))_{x=v=1}$ \\
Second Zagreb & $\left(D_{x} D_{y}\right)(M(G ; x, y))_{x=v=1}$ \\
Second Modified Zagreb & $\left(S_{x} S_{y}\right)(M(G ; x, y))_{x=v=1}$ \\
Inverse Randić & $\left(D_{x}^{\alpha} D_{y}^{\alpha}\right)(\mathrm{M}(\mathrm{G} ; \mathrm{x}, \mathrm{y}))_{x=y=1}$ \\
General Randić & $\left(S_{x}^{\alpha} S_{y}^{\alpha}\right)(M(G ; x, y))_{x=y=1}$ \\
Symmetric Division Index & $\left(D_{x} S_{v}+S_{x} D_{y}\right)(M(G ; x, y))_{x=v=1}$ \\
Harmonic Index & $\left.2 S_{x} J(M(G ; x, y))_{x=1}(G ; x, y)\right)_{x=1}$ \\
Inverse sum Index & $S_{x} J D_{x} D_{y}\left(M\left(G ; D_{x}^{3} D_{y}^{3}(M(G ; x, y))_{x=1}\right.\right.$ \\
Augmented Zagreb Index & $S_{x}^{3} Q_{-2} J D_{x}^{3}(M$ \\
\hline
\end{tabular}


Where $D_{x}=x \frac{\partial f(x, y)}{\partial x}, D_{y}=y \frac{\partial f(x, y)}{\partial y}, S_{x}=\int_{0}^{x} \frac{f(t, y)}{t} d t$,

$$
\begin{gathered}
S_{y}=\int_{0}^{y} \frac{f(x, t)}{t} d t \\
J(f(x, y))=f(x, x), Q_{\alpha}(f(x, y))=x^{\alpha} f(x, y) .
\end{gathered}
$$

In 2013, Shirdel et al. in [29] proposed "hyper-Zagreb index" which is also a degree based index.

Definition 2.2. Let $\mathrm{G}$ be a simple connected graph. Then the hyper-Zagreb index of $\mathrm{G}$ is defined as:

$$
H M(\mathrm{G})=\sum_{u v \in \mathrm{E}(\mathrm{G})}\left[d_{u}+d_{v}\right]^{2} .
$$

In 2012, Ghorbani and Azimi [30] proposed two new variants of Zagreb indices.

Definition 2.3. Let $\mathrm{G}$ be a simple connected graph. Then the first multiple Zagreb index of $\mathrm{G}$ is defined as:

$$
P M_{1}(\mathrm{G})=\prod_{u v \in \mathrm{E}(\mathrm{G})}\left[d_{u}+d_{v}\right] .
$$

Definition 2.4. Let $G$ be a simple connected graph. Then the second multiple Zagreb index of $\mathrm{G}$ is defined as:

$$
P M_{2}(\mathrm{G})=\prod_{u v \in \mathrm{E}(\mathrm{G})}\left[d_{u} \cdot d_{v}\right] .
$$

Definition 2.5. Let $\mathrm{G}$ be a simple connected graph. Then the first Zagreb polynomial of $\mathrm{G}$ is defined as:

$$
M_{1}(\mathrm{G}, \mathrm{x})=\sum_{u v \in \mathrm{E}(\mathrm{G})} x^{\left[d_{u}+d_{v}\right]} .
$$

Definition 2.6. Let $\mathrm{G}$ be a simple connected graph. Then the second Zagreb polynomial of $G$ is defined as:

$$
M_{2}(\mathrm{G}, \mathrm{x})=\sum_{u v \in \mathrm{E}(\mathrm{G})} x^{\left[d_{u} \cdot d_{v}\right]} .
$$

Definition 2.7. Let $\mathrm{G}$ be a simple connected graph. Then the Forgotten polynomial of $\mathrm{G}$ is defined as:

$$
F(\mathrm{G}, \mathrm{x})=\sum_{u v \in \mathrm{E}(\mathrm{G})} x^{\left[\left(d_{u}\right)^{2}+\left(d_{v}\right)^{2}\right]} .
$$

Definition 2.8. Let $\mathrm{G}$ be a simple connected graph. Then the forgotten index of $G$ is defined as:

$$
F(\mathrm{G})=\sum_{u v \in \mathrm{E}(\mathrm{G})}\left[\left(d_{u}\right)^{2}+\left(d_{v}\right)^{2}\right]
$$

In [31] we previously found some indices and polynomial for the Cartesian product of two paths $P_{n} P_{m}$ in.

\section{Main Results and Discussions}

In this paper we will compute the M-polynomial, first and second Zagreb polynomials and forgotten polynomial for the Cartesian Product of a Path and a complete bipartite graph $P_{n} K_{(1, m)}$ for all values of $\mathrm{n}$ and $m$. From the M-polynomial, we will compute many degree-based topological indices such that general Randić index, inverse Randić index, first and second Zagreb index, modified Zagreb index, Symmetric division index, Inverse sum index augmented Zagreb index and harmonic index for $P_{n} K_{(1, m)}$. Also, We will compute the hyper- Zagreb index, the first and second multiple Zagreb index and the forgotten index for $P_{n} K_{(1, m)}$.

Theorem 3.1. The M-polynomial for $P_{n} K_{(1, m)}$ is:

$$
\begin{aligned}
& M\left(P_{n} K_{(1, m)}, x, y\right)=2 m x^{2} y^{3}+m(n-3) x^{3} y^{3}+2 m x^{2} y^{m+1} \\
& \quad+m(n-2) x^{3} y^{m+2}+2 x^{m+1} y^{m+2}+(n-3) x^{m+2} y^{m+2} .
\end{aligned}
$$

Proof. Clearly, we have $\left|V\left(P_{n} K_{(1, m)}\right)\right|=n(m+1)$.

$$
\left|E\left(P_{n} K_{(1, m)}\right)\right|=2 n m+n-m-1 .
$$

We can divide the edge set into following four partitions:

$$
\begin{gathered}
E_{1}\left(P_{n} K_{(1, m)}\right)=\left\{e=u v \in E\left(P_{n} K_{(1, m)}\right): d_{u}=2, d_{v}=3\right\} . \\
E_{2}\left(P_{n} K_{(1, m)}\right)=\left\{e=u v \in E\left(P_{n} K_{(1, m)}\right): d_{u}=3, d_{v}=3\right\} . \\
E_{3}\left(P_{n} K_{(1, m)}\right)=\left\{e=u v \in E\left(P_{n} K_{(1, m)}\right): d_{u}=2, d_{v}=m+1\right\} . \\
E_{4}\left(P_{n} K_{(1, m)}\right)=\left\{e=u v \in E\left(P_{n} K_{(1, m)}\right): d_{u}=3, d_{v}=m+2\right\} . \\
E_{5}\left(P_{n} K_{(1, m)}\right)=\left\{e=u v \in E\left(P_{n} K_{(1, m)}\right): d_{u}=m+1, d_{v}=m+2\right\} . \\
E_{6}\left(P_{n} K_{(1, m)}\right)=\left\{e=u v \in E\left(P_{n} K_{(1, m)}\right): d_{u}=m+2, d_{v}=m+2\right\} \\
\left|E_{1}\left(P_{n} K_{(1, m)}\right)\right|=2 m,\left|E_{2}\left(P_{n} K_{(1, m)}\right)\right|=m(n-3), \\
\left|E_{3}\left(P_{n} K_{(1, m)}\right)\right|=2 m,\left|E_{4}\left(P_{n} K_{(1, m)}\right)\right|=m(n-2),
\end{gathered}
$$




$$
\left|E_{5}\left(P_{n} K_{(1, m)}\right)\right|=2,\left|E_{6}\left(P_{n} K_{(1, m)}\right)\right|=n-3 .
$$

Now, by definition of M-polynomial, we have:メ

$$
\begin{gathered}
M\left(P_{n} K_{(1, m)}, x, y\right)=\sum_{\delta \leq i \leq j \leq \Delta} m_{i, j} x^{i} y^{j} \\
=\sum_{2 \leq 3} m_{2,3} x^{2} y^{3}+\sum_{3 \leq 3} m_{3,3} x^{3} y^{3}+\sum_{2 \leq m+1} m_{2, m+1} x^{2} y^{m+1} \\
+\sum_{3 \leq m+2} m_{3, m+2} x^{3} y^{m+2}+\sum_{m+1 \leq m+2} m_{m+1, m+2} x^{m+1} y^{m+2}+\sum_{m+2 \leq m+2} m_{m+2, m+2} x^{m+2} y^{m+2} \\
=\sum_{u, v \in E_{1}\left(P_{n} K_{(1, m)}\right)} m_{2,3} x^{2} y^{3}+\sum_{u, v \in E_{2}\left(P_{n} K_{(1, m)}\right)} m_{3,3} x^{3} y^{3} \\
+\sum_{u, v \in E_{3}\left(P_{n} K_{(1, m)}\right)} m_{2, m+1} x^{2} y^{m+1}+\sum_{u, v \in E_{4}\left(P_{n} K_{(1, m)}\right)} m_{3, m+2} x^{3} y^{m+2} \\
\sum_{u, v \in E_{5}\left(P_{n} K_{(1, m)}\right)} m_{m+1, m+2} x^{m+1} y^{m+2}+\sum_{u, v \in E_{6}\left(P_{n} K_{(1, m)}\right)} m_{m+2, m+2} x^{m+2} y^{m+2} \\
=\left|E_{1}\left(P_{n} K_{(1, m)}\right)\right| x^{2} y^{3}+\left|E_{2}\left(P_{n} K_{(1, m)}\right)\right| x^{3} y^{3}+\left|E_{3}\left(P_{n} K_{(1, m)}\right)\right| x^{2} y^{m+1}+\left|E_{4}\left(P_{n} K_{(1, m)}\right)\right| x^{3} y^{m+2} \\
+\left|E_{5}\left(P_{n} K_{(1, m)}\right)\right| x^{m+1} y^{m+2}+\left|E_{6}\left(P_{n} K_{(1, m)}\right)\right| x^{m+2} y^{m+2} \\
=2 m x^{2} y^{3}+m(n-3) x^{3} y^{3}+2 m x^{2} y^{m+1}+m(n-2) x^{3} y^{m+2}+2 x^{m+1} y^{m+2}+(n-3) x^{m+2} y^{m+2} .
\end{gathered}
$$

Now, we compute some degree-based topological indices of the Cartesian product of a Path and a complete bipartite graph $P_{n} K_{(1, m)}$ for all values of $\mathrm{n}$ from this M-polynomial.

Theorem 3.2.

1) $M_{1}\left(P_{n} K_{(1, m)}\right)=13 n m-14 m+4 n+m^{2} n-6$.

2) $M_{2}\left(P_{n} K_{(1, m)}\right)=-3 m^{2}+19 n m+4 n m^{2}-29 m+4 n-8$.

3) ${ }^{\mathrm{m}} M_{2}\left(P_{n} K_{(1, m)}\right)=\left(\frac{1}{9}+\frac{1}{3(m+2)}\right) n m+\frac{m}{m+1}-\frac{2 m}{3(m+2)}+\frac{2}{(m+1)(m+2)}+\frac{n-3}{(m+2)^{2}}$.

4) $\left.R R_{\alpha}\left(P_{n} K_{(1, m)}\right)=m\left(2 \times 6^{\alpha}+9^{\alpha}(n-2)+2(2 m+2)^{\alpha}\right)+(3 m+6)^{\alpha}(n-2)\right)+2\left(m^{2}+3 m+2\right)^{\alpha}+(n-$ 3) $(m+2)^{2 \alpha}$.

5) $R_{\alpha}\left(P_{n} K_{(1, m)}\right)=m\left(\frac{2}{6^{\alpha}}+\frac{n-3}{9^{\alpha}}+\frac{2}{(2 m+2)^{\alpha}}+\frac{n-2}{(3 m+6)^{\alpha}}\right)+\frac{2}{\left(m^{2}+3 m+2\right)^{\alpha}}+\frac{n-3}{(m+2)^{2 \alpha}}$.

6) $S D D\left(P_{n} K_{(1, m)}\right)=m\left(\frac{13}{3}+2(n-3)+\frac{4}{m+1}+m+1\right)+\frac{3 m}{m+2}(n-2)+\frac{m}{3}(m+2)(n-2)+\frac{2(m+1)}{m+2}+\frac{2(m+2)}{m+1}+$ $2(n-3)$.

7) $H\left(P_{n} K_{(1, m)}\right)=m\left(\frac{4}{5}+\frac{n-3}{3}+\frac{4}{m+3}+\frac{2(n-2)}{m+5}\right)+\frac{4}{2 m+3}+\frac{2(n-3)}{2 m+4}$.

8) $I\left(P_{n} K_{(1, m)}\right)=m\left(\frac{12}{5}+\frac{3(n-3)}{2}+\frac{4(m+1)}{m+3}+\frac{3(n-2)(m+2)}{m+5}\right)+\frac{2(m+1)(m+2)}{2 m+3}+\frac{(n-3)(m+2)}{2}$.

9) $A\left(P_{n} K_{(1, m)}\right)=m\left(32+\frac{729}{64}(n-3)+\frac{27}{(m+3)^{3}}(m+2)^{3}(n-2)\right)+\frac{2(m+1)^{3}(m+2)^{3}}{(2 m+1)^{3}}+\frac{m-3}{(2 m+2)^{3}}(m+2)^{6}$.

Proof. Let $M\left(P_{n} K_{(1, m)}, x, y\right)=f(x, y)=2 m x^{2} y^{3}+m(n-3) x^{3} y^{3}+2 m x^{2} y^{m+1}+m(n-2) x^{3} y^{m+2}+2 x^{m+1} y^{m+2}+$ $(n-3) x^{m+2} y^{m+2}$.

Then:

$$
\begin{gathered}
D_{x} f(x, y)=4 m x^{2} y^{3}+3 m(n-3) x^{3} y^{3}+4 m x^{2} y^{m+1}+3 m(n-2) x^{3} y^{m+2} \\
+2(m+1) x^{m+1} y^{m+2}+(n-3)(m+2) x^{m+2} y^{m+2} . \\
D_{y} f(x, y)=6 m x^{2} y^{3}+3 m(n-3) x^{3} y^{3}+2 m(m+1) x^{2} y^{m+1} \\
+m(m+2)(n-2) x^{3} y^{m+2}+2(m+2) x^{m+1} y^{m+2}+(n-3)(m+2) x^{m+2} y^{m+2} \\
D_{x} D_{y} f(x, y)=12 m x^{2} y^{3}+9 m(n-3) x^{3} y^{3}+4 m(m+1) x^{2} y^{m+1} \\
+3 m(m+2)(n-2) x^{3} y^{m+2}+2(m+1)(m+2) x^{m+1} y^{m+2}+(n-3)(m+2)^{2} x^{m+2} y^{m+2}
\end{gathered}
$$




$$
\begin{aligned}
& S_{y} f(x, y)=\frac{2}{3} m x^{2} y^{3}+\frac{m}{3}(n-3) x^{3} y^{3}+\frac{2}{m+1} m x^{2} y^{m+1} \\
& +\frac{m}{m+2}(n-2) x^{3} y^{m+2}+\frac{2}{m+2} x^{m+1} y^{m+2}+\frac{n-3}{m+2} x^{m+2} y^{m+2} \text {. } \\
& S_{x} S_{y} f(x, y)=\frac{1}{3} m x^{2} y^{3}+\frac{m}{9}(n-3) x^{3} y^{3}+\frac{1}{m+1} m x^{2} y^{m+1} \\
& +\frac{m}{3(m+2)}(n-2) x^{3} y^{m+2}+\frac{2}{(m+1)(m+2)} x^{m+1} y^{m+2}+\frac{n-3}{(m+2)^{2}} x^{m+2} y^{m+2} \text {. } \\
& S_{y}^{\alpha} f(x, y)=\frac{2}{3^{\alpha}} m x^{2} y^{3}+\frac{m}{3^{\alpha}}(n-3) x^{3} y^{3}+\frac{2}{(m+1)^{\alpha}} m x^{2} y^{m+1} \\
& +\frac{m}{(m+2)^{\alpha}}(n-2) x^{3} y^{m+2}+\frac{2}{(m+2)^{\alpha}} x^{m+1} y^{m+2}+\frac{n-3}{(m+2)^{\alpha}} x^{m+2} y^{m+2} \text {. } \\
& S_{x}^{\alpha} S_{y}^{\alpha} f(x, y)=\frac{2}{6^{\alpha}} m x^{2} y^{3}+\frac{m}{9^{\alpha}}(n-3) x^{3} y^{3}+\frac{2}{(2 m+2)^{\alpha}} m x^{2} y^{m+1} \\
& +\frac{m}{(3 m+6)^{\alpha}}(n-2) x^{3} y^{m+2}+\frac{2}{(m+1)^{\alpha}(m+2)^{\alpha}} x^{m+1} y^{m+2}+\frac{n-3}{(m+2)^{2 \alpha}} x^{m+2} y^{m+2} \text {. } \\
& D_{y}^{\alpha} f(x, y)=2 \times 3^{\alpha} m x^{2} y^{3}+3^{\alpha} m(n-3) x^{3} y^{3}+2 m(m+1)^{\alpha} x^{2} y^{m+1} \text {. } \\
& +m(m+2)^{\alpha}(n-2) x^{3} y^{m+2}+2(m+2)^{\alpha} x^{m+1} y^{m+2}+(n-3)(m+2)^{\alpha} x^{m+2} y^{m+2} \text {. } \\
& D_{x}^{\alpha} D_{y}^{\alpha} f(x, y)=2 \times 6^{\alpha} m x^{2} y^{3}+9^{\alpha} m(n-3) x^{3} y^{3}+2 m(2 m+2)^{\alpha} x^{2} y^{m+1} \text {. } \\
& +m(3 m+6)^{\alpha}(n-2) x^{3} y^{m+2}+2(m+1)^{\alpha}(m+2)^{\alpha} x^{m+1} y^{m+2}+(n-3)(m+2)^{2 \alpha} x^{m+2} y^{m+2} \text {. } \\
& D_{x} S_{y} f(x, y)=\frac{4}{3} m x^{2} y^{3}+m(n-3) x^{3} y^{3}+\frac{4}{m+1} m x^{2} y^{m+1} \\
& +\frac{3 m}{m+2}(n-2) x^{3} y^{m+2}+\frac{2(m+1)}{m+2} x^{m+1} y^{m+2}+(n-3) x^{m+2} y^{m+2} \text {. } \\
& S_{x} D_{y} f(x, y)=3 m x^{2} y^{3}+m(n-3) x^{3} y^{3}+m(m+1) x^{2} y^{m+1} \\
& +\frac{m}{3}(m+2)(n-2) x^{3} y^{m+2}+\frac{2(m+2)}{m+1} x^{m+1} y^{m+2}+(n-3) x^{m+2} y^{m+2} \text {. } \\
& J f(x, y)=2 m x^{5}+m(n-3) x^{6}+2 m x^{m+3}+m(n-2) x^{m+5}+2 x^{2 m+3}+(n-3) x^{2 m+4} . \\
& S_{x} J f(x, y)=\frac{2}{5} m x^{5}+\frac{m}{6}(n-3) x^{6}+\frac{2 m}{m+3} x^{m+3}+\frac{m(n-2)}{m+5} x^{m+5}+\frac{2}{2 m+3} x^{2 m+3}+\frac{n-3}{2 m+4} x^{2 m+4} \text {. } \\
& J D_{x} D_{y} f(x, y)=12 m x^{5}+9 m(n-3) x^{6}+4 m(m+1) x^{m+3} \\
& +3 m(m+2)(n-2) x^{m+5}+2(m+1)(m+2) x^{2 m+3}+(n-3)(m+2)^{2} x^{2 m+4} \text {. } \\
& S_{x} J D_{x} D_{y} f(x, y)=\frac{12}{5} m x^{5}+\frac{3}{2} m(n-3) x^{6}+\frac{4 m(m+1)}{m+3} x^{m+3} \\
& +\frac{3 m(m+2)(n-2)}{m+5} x^{m+5}+\frac{2(m+1)(m+2)}{2 m+3} x^{2 m+3}+\frac{(n-3)(m+2)}{2} x^{2 m+4} \text {. } \\
& D_{y}^{3} f(x, y)=54 m x^{2} y^{3}+27 m(n-3) x^{3} y^{3}+2 m(m+1)^{3} x^{2} y^{m+1} \\
& +m(m+2)^{3}(n-2) x^{3} y^{m+2}+2(m+2)^{3} x^{m+1} y^{m+2}+(n-3)(m+2)^{3} x^{m+2} y^{m+2} \text {. } \\
& D_{x}^{3} D_{y}^{3} f(x, y)=432 m x^{2} y^{3}+729 m(n-3) x^{3} y^{3}+16 m(m+1)^{3} x^{2} y^{m+1} \\
& +27 m(m+2)^{3}(n-2) x^{3} y^{m+2}+2(m+1)^{3}(m+2)^{3} x^{m+1} y^{m+2}+(n-3)(m+2)^{6} x^{m+2} y^{m+2} \text {. }
\end{aligned}
$$




$$
\begin{gathered}
J D_{x}^{3} D_{y}^{3} f(x, y)=432 m x^{5}+729 m(n-3) x^{6}+16 m(m+1)^{3} x^{m+3} \\
+27 m(m+2)^{3}(n-2) x^{m+5}+2(m+1)^{3}(m+2)^{3} x^{2 m+3}+(n-3)(m+2)^{6} x^{2 m+4} . \\
Q_{-2} J D_{x}^{3} D_{y}^{3} f(x, y)=432 m x^{3}+729 m(n-3) x^{4}+16 m(m+1)^{3} x^{m+1} \\
+27 m(m+2)^{3}(n-2) x^{m+3}+2(m+1)^{3}(m+2)^{3} x^{2 m+1}+(n-3)(m+2)^{6} x^{2 m+2} . \\
S_{x}^{3} Q_{-2} J D_{x}^{3} D_{y}^{3} f(x, y)=16 m x^{3}+\frac{729}{64} m(n-3) x^{4}+16 m x^{m+1} \\
+\frac{27}{(m+3)^{3}} m(m+2)^{3}(n-2) x^{m+3} \cdot+\frac{2}{(2 m+1)^{3}}(m+1)^{3}(m+2)^{3} x^{2 m+1}+\frac{n-3}{(2 m+2)^{3}}(m+2)^{6} x^{2 m+2} .
\end{gathered}
$$

Now, from Table 1:

1. First Zagreb Index

$$
M_{1}\left(P_{n} K_{(1, m)}\right)=\left.\left(D_{x}+D_{y}\right) f(x, y)\right|_{x=y=1}=13 n m-14 m+4 n+m^{2} n-6 .
$$

2. Second Zagreb Index

$$
M_{2}\left(P_{n} K_{(1, m)}\right)=\left.\left(D_{x} D_{y}\right)(f(x, y))\right|_{x=y=1}-8
$$

3. Modified second Zagreb Index

$$
\begin{gathered}
\mathrm{m}_{M_{2}}\left(P_{n} K_{(1, m)}\right)=\left.\left(S_{x} S_{y}\right)(f(x, y))\right|_{x=y=1}=-3 m^{2}+19 n m+4 n m^{2}-29 m+4 n \\
=\left(\frac{1}{9}+\frac{1}{3(m+2)}\right) n m+\frac{m}{m+1}-\frac{2 m}{3(m+2)}+\frac{2}{(m+1)(m+2)}+\frac{n-3}{(m+2)^{2}} .
\end{gathered}
$$

4. Inverse Randic Index

$$
\begin{gathered}
R R_{\alpha}\left(P_{n} K_{(1, m)}\right)=\left.\left(D_{x}^{\alpha} D_{y}^{\alpha}\right) f(x, y)\right|_{x=y=1} \\
=m\left(2 \times 6^{\alpha}+9^{\alpha}(n-2)+2(2 m+2)^{\alpha}+(3 m+6)^{\alpha}(n-2)\right)+2\left(m^{2}+3 m+2\right)^{\alpha}+(n-3)(m+2)^{2 \alpha} .
\end{gathered}
$$

5. Generalized Randic Index

$$
\begin{gathered}
R_{\alpha}\left(P_{n} K_{(1, m)}\right)=\left.\left(S_{x}^{\alpha} S_{y}^{\alpha}\right)(f(x, y))\right|_{x=y=1} \\
=m\left(\frac{2}{6^{\alpha}}+\frac{n-3}{9^{\alpha}}+\frac{2}{(2 m+2)^{\alpha}}+\frac{n-2}{(3 m+6)^{\alpha}}\right)+\frac{2}{\left(m^{2}+3 m+2\right)^{\alpha}}+\frac{n-3}{(m+2)^{2 \alpha}} .
\end{gathered}
$$

6. Symmetric Division Index

$$
\begin{gathered}
\quad \operatorname{SDD}\left(P_{n} K_{(1, m)}\right)=\left.\left(D_{x} S_{y}+S_{x} D_{y}\right)(f(x, y))\right|_{x=y=1} \\
=m\left(\frac{13}{3}+2(n-3)+\frac{4}{m+1}+m+1\right)+\frac{3 m}{m+2}(n-2)+\frac{m}{3}(m+2)(n-2)+\frac{2(m+1)}{m+2}+\frac{2(m+2)}{m+1}+2(n-3) .
\end{gathered}
$$

7. Harmonic Index

$$
H\left(P_{n} K_{(1, m)}\right)=\left.2 S_{x} J(f(x, y))\right|_{x=1}=m\left(\frac{4}{5}+\frac{n-3}{3}+\frac{4}{m+3}+\frac{2(n-2)}{m+5}\right)+\frac{4}{2 m+3}+\frac{2(n-3)}{2 m+4} .
$$

8. Inverse Sum Index

$$
\begin{gathered}
I\left(P_{n} K_{(1, m)}\right)=\left.S_{x} J D_{x} D_{y}(f(x, y))\right|_{x=1} \\
=m\left(\frac{12}{5}+\frac{3(n-3)}{2}+\frac{4(m+1)}{m+3}+\frac{3(n-2)(m+2)}{m+5}\right)+\frac{2(m+1)(m+2)}{2 m+3}+\frac{(n-3)(m+2)}{2} .
\end{gathered}
$$


9. Augmented Zagreb Index

$$
\begin{gathered}
A\left(P_{n} K_{(1, m)}\right)=\left.\left(S_{x}^{3} Q_{-2} J D_{x}^{3} D_{y}^{3}\right)(f(x, y))\right|_{x=1} \\
=m\left(32+\frac{729}{64}(n-3)+\frac{27}{(m+3)^{3}}(m+2)^{3}(n-2)\right)+\frac{2(m+1)^{3}(m+2)^{3}}{(2 m+1)^{3}}+\frac{m-3}{(2 m+2)^{3}}(m+2)^{6} .
\end{gathered}
$$

Theorem 3.3 The hyper-Zagreb index of $P_{n} K_{(1, m)}$ is:

$$
H M\left(P_{n} K_{(1, m)}\right)=50 m+36 m(n-2)+2 m(m+3)^{2}+m(n-2)(m+5)^{2}+2(2 m+3)^{2}+(n-3)(2 m+4)^{2} .
$$

Proof. By the definition of the hyper-Zagreb index

$$
\begin{gathered}
H M\left(P_{n} K_{(1, m)}\right)=\sum_{u v \in E\left(P_{n} K_{(1, m)}\right)}\left[d_{u}+d_{v}\right]^{2} \\
=\sum_{u v \in E_{1}\left(P_{n} K_{(1, m)}\right)}\left[d_{u}+d_{v}\right]^{2}+\sum_{u v \in E_{2}\left(P_{n} K_{(1, m)}\right)}\left[d_{u}+d_{v}\right]^{2}+\sum_{u v \in E_{3}\left(P_{n} K_{(1, m)}\right)}\left[d_{u}+d_{v}\right]^{2}+\sum_{u v \in E_{4}\left(P_{n} K_{(1, m)}\right)}\left[d_{u}+d_{v}\right]^{2} \\
+\sum_{u v \in E_{5}\left(P_{n} K_{(1, m)}\right)}\left[d_{u}+d_{v}\right]^{2}+\sum_{u v \in E_{6}\left(P_{n} K_{(1, m)}\right)}\left[d_{u}+d_{v}\right]^{2} \\
=\left|E_{1}\left(P_{n} K_{(1, m)}\right)\right|[2+3]^{2}+\left|E_{2}\left(P_{n} K_{(1, m)}\right)\right|[3+3]^{2}+\left|E_{3}\left(P_{n} K_{(1, m)}\right)\right|[m+3]^{2}+\left|E_{4}\left(P_{n} K_{(1, m)}\right)\right|[m+ \\
5]^{2}+\left|E_{5}\left(P_{n} K_{(1, m)}\right)\right|[2 m+3]^{2}+\left|E_{6}\left(P_{n} K_{(1, m)}\right)\right|[2 m+4]^{2} \\
=50 m+36 m(n-2)+2 m(m+3)^{2}+m(n-2)(m+5)^{2}+2(2 m+3)^{2}+(n-3)(2 m+4)^{2} .
\end{gathered}
$$

Theorem 3.4. The first multiple Zagreb index of $P_{n} K_{(1, m)}$ is:

$$
P M_{1}\left(P_{n} K_{(1, m)}\right)=5^{2 m} \times 6^{m(n-2)} \times(m+3)^{2 m} \times(m+5)^{m(n-2)} \times(2 m+3)^{2} \times(2 m+4)^{n-3} .
$$

Proof. By the definition of the first multiple Zagreb index:

$$
\begin{gathered}
P M_{1}\left(P_{n} K_{(1, m)}\right)=\prod_{u v \in \mathrm{E}\left(P_{n} K_{(1, m))}\right.}\left[d_{u}+d_{v}\right]=\prod_{u v \in E_{1}\left(P_{n} K_{(1, m)}\right)}\left[d_{u}+d_{v}\right] \times \prod_{u v \in E_{2}\left(P_{n} K_{(1, m)}\right)}\left[d_{u}+d_{v}\right] \\
+\prod_{u v \in E_{3}\left(P_{n} K_{(1, m)}\right)}\left[d_{u}+d_{v}\right] \times \prod_{u v \in E_{4}\left(P_{n} K_{(1, m)}\right)}\left[d_{u}+d_{v}\right]+\prod_{u v \in E_{5}\left(P_{n} K_{(1, m)}\right)}\left[d_{u}+d_{v}\right] \times \prod_{u v \in E_{6}\left(P_{n} K_{(1, m)}\right)}\left[d_{u}+d_{v}\right] \\
=[2+3]^{\left|E_{1}\left(P_{n} K_{(1, m)}\right)\right|} \times[3+3]^{\left|E_{2}\left(P_{n} K_{(1, m)}\right)\right|}+[m+3]^{\left|E_{3}\left(P_{n} K_{(1, m)}\right)\right|} \times[m+5]^{\mid E_{4}\left(P_{n} K_{(1, m))} \mid\right.} \\
+[2 m+3]^{\left|E_{5}\left(P_{n} K_{(1, m)}\right)\right|} \times[2 m+4]^{\left|E_{6}\left(P_{n} P_{m}\right)\right|} \\
=5^{2 m} \times 6^{m(n-2)} \times(m+3)^{2 m} \times(m+5)^{m(n-2)} \times(2 m+3)^{2} \times(2 m+4)^{n-3} .
\end{gathered}
$$

Theorem 3.5. The second multiple Zagreb index of $P_{n} K_{(1, m)}$ is:

$$
\begin{gathered}
\mathrm{PM}_{2}\left(P_{n} K_{(1, m)}\right)=6^{2 m} \times 9^{m(n-2)} \times(2 m+2)^{2 m} \times(3 m+6)^{m(n-2)} \\
\times\left(m^{2}+3 m+2\right)^{2} \times\left(m^{2}+4 m+4\right)^{n-3} .
\end{gathered}
$$

Proof. By the definition of the second multiple Zagreb index:

$$
\begin{gathered}
P M_{2}\left(P_{n} K_{(1, m)}\right)=\prod_{u v \in \mathrm{E}\left(P_{n}\right.}\left[d_{(1, m)} \times d_{v}\right]=\prod_{u v \in E_{1}\left(P_{n} K_{(1, m))}\right.}\left[d_{u} \times d_{v}\right] \times \prod_{u v \in E_{2}\left(P_{n} K_{(1, m))}\right.}\left[d_{u} \times d_{v}\right] \\
\times \prod_{u v \in E_{3}\left(P_{n} K_{(1, m)}\right)}\left[d_{u} \times d_{v}\right] \times \prod_{u v \in E_{4}\left(P_{n} K_{(1, m)}\right)}\left[d_{u} \times d_{v}\right] \times \prod_{u v \in E_{5}\left(P_{n} K_{(1, m)}\right)}\left[d_{u} \times d_{v}\right] \times \prod_{u v \in E_{6}\left(P_{n} K_{(1, m)}\right)}\left[d_{u} \times d_{v}\right]
\end{gathered}
$$




$$
\begin{gathered}
=[2 \times 3]^{\left|E_{1}\left(P_{n} K_{(1, m)}\right)\right|} \times[3 \times 3]^{\left|E_{2}\left(P_{n} K_{(1, m)}\right)\right|} \times[2(m+1)]^{\left|E_{3}\left(P_{n} K_{(1, m)}\right)\right|} \times[3(m+2)]^{\left|E_{4}\left(P_{n} K_{(1, m)}\right)\right|} \\
\times[(m+1)(m+2)]^{\left|E_{5}\left(P_{n} K_{(1, m)}\right)\right|} \times[(m+2)(m+2)]^{\left|E_{6}\left(P_{n} K_{(1, m)}\right)\right|} \\
=6^{2 m} \times 9^{m(n-2)} \times(2 m+2)^{2 m} \times(3 m+6)^{m(n-2)} \times\left(m^{2}+3 m+2\right)^{2} \times\left(m^{2}+4 m+4\right)^{n-3} .
\end{gathered}
$$

Theorem 3.6. The first Zagreb polynomial of $P_{n} K_{(1, m)}$ is:

$$
M_{1}\left(P_{n} K_{(1, m)}, x\right)=2 m x^{5}+m(n-3) x^{6}+2 m x^{m+3}+m(n-2) x^{m+5}+2 x^{2 m+3}+(n-3) x^{2 m+4} .
$$

Proof. By the definition of the first Zagreb polynomial:

$$
\begin{gathered}
M_{1}\left(P_{n} K_{(1, m)}, x\right)=\sum_{u v \in \mathrm{E}\left(P_{n} K_{(1, m)}\right.} x^{\left[d_{u}+d_{v}\right]}=\sum_{u v \in E_{1}\left(P_{n} K_{(1, m)}\right)} x^{\left[d_{u}+d_{v}\right]}+\sum_{u v \in E_{2}\left(P_{n} K_{(1, m)}\right)} x^{\left[d_{u}+d_{v}\right]} \\
+\sum_{u v \in E_{3}\left(P_{n} K_{(1, m)}\right)} x^{\left[d_{u}+d_{v}\right]}+\sum_{u v \in E_{4}\left(P_{n} K_{(1, m)}\right)} x^{\left[d_{u}+d_{v}\right]}+\sum_{u v \in E_{5}\left(P_{n} K_{(1, m)}\right)} x^{\left[d_{u}+d_{v}\right]}+\sum_{u v \in E_{6}\left(P_{n} K_{(1, m)}\right)} x^{\left[d_{u}+d_{v}\right]} \\
=\left|E_{1}\left(P_{n} K_{(1, m)}\right)\right| x^{5}+\left|E_{2}\left(P_{n} K_{(1, m)}\right)\right| x^{6}+\left|E_{3}\left(P_{n} K_{(1, m)}\right)\right| x^{m+3}+\left|E_{4}\left(P_{n} K_{(1, m)}\right)\right| x^{m+5} \\
\quad+\left|E_{5}\left(P_{n} K_{(1, m)}\right)\right| x^{2 m+3}+\left|E_{6}\left(P_{n} K_{(1, m)}\right)\right| x^{2 m+4} \\
=2 m x^{5}+m(n-3) x^{6}+2 m x^{m+3}+m(n-2) x^{m+5}+2 x^{2 m+3}+(n-3) x^{2 m+4} .
\end{gathered}
$$

Theorem 3.7. The Then second Zagreb polynomial of $P_{n} K_{(1, m)}$ is:

$$
M_{2}\left(P_{n} K_{(1, m)}, x\right)=2 m x^{6}+m(n-3) x^{9}+2 m x^{2 m+2}+m(n-2) x^{3 m+6}+2 x^{m^{2}+3 m+2}+(n-3) x^{m^{2}+4 m+4} .
$$

Proof. By the definition of the second Zagreb polynomial:

$$
\begin{gathered}
M_{2}\left(P_{n} K_{(1, m)}, x\right)=\sum_{u v \in E\left(P_{n} K_{(1, m)}\right.} x^{\left[d_{u} \times d_{v}\right]}=\sum_{u v \in E_{1}\left(P_{n} K_{(1, m)}\right)} x^{\left[d_{u} \times d_{v}\right]}+\sum_{u v \in E_{2}\left(P_{n} K_{(1, m)}\right)} x^{\left[d_{u} \times d_{v}\right]} \\
+\sum_{u v \in E_{3}\left(P_{n} K_{(1, m)}\right)} x^{\left[d_{u} \times d_{v}\right]}+\sum_{u v \in E_{4}\left(P_{n} K_{(1, m)}\right)} x^{\left[d_{u} \times d_{v}\right]}+\sum_{u v \in E_{5}\left(P_{n} K_{(1, m)}\right)} x^{\left[d_{u} \times d_{v}\right]}+\sum_{u v \in E_{6}\left(P_{n} K_{(1, m)}\right)} x^{\left[d_{u} \times d_{v}\right]} \\
=\left|E_{1}\left(P_{n} K_{(1, m)}\right)\right| x^{6}+\left|E_{2}\left(P_{n} K_{(1, m)}\right)\right| x^{9}+\left|E_{3}\left(P_{n} K_{(1, m)}\right)\right| x^{2 m+2}+\left|E_{4}\left(P_{n} K_{(1, m)}\right)\right| x^{3 m+6} \\
+\left|E_{5}\left(P_{n} K_{(1, m)}\right)\right| x^{m^{2}+3 m+2}+\left|E_{6}\left(P_{n} K_{(1, m)}\right)\right| x^{m^{2}+4 m+4} \\
=2 m x^{6}+m(n-3) x^{9}+2 m x^{2 m+2}+m(n-2) x^{3 m+6}+2 x^{m^{2}+3 m+2}+(n-3) x^{m^{2}+4 m+4} .
\end{gathered}
$$

Theorem 3.8. The forgotten polynomial of $P_{n} K_{(1, m)}$ is:

$$
\begin{gathered}
F\left(P_{n} K_{(1, m)}\right)=2 m x^{13}+m(n-3) x^{18}+2 m x^{4+(m+1)^{2}} \\
+m(n-2) x^{9+(m+2)^{2}}+2 x^{(m+1)^{2}+(m+2)^{2}}+(n-3) x^{2(m+2)^{2}} .
\end{gathered}
$$

Proof. By the definition of the forgotten polynomial:

$$
\begin{gathered}
F\left(P_{n} K_{(1, m)}, x\right)=\sum_{u v \in E\left(P_{n} K_{(1, m)}\right)} x^{\left[\left(d_{u}\right)^{2}+\left(d_{v}\right)^{2}\right]}=\sum_{u v \in E_{1}\left(P_{n} K_{(1, m)}\right)} x^{\left[\left(d_{u}\right)^{2}+\left(d_{v}\right)^{2}\right]}+\sum_{u v \in E_{2}\left(P_{n} K_{(1, m)}\right)} x^{\left[\left(d_{u}\right)^{2}+\left(d_{v}\right)^{2}\right]} \\
+\sum_{u v \in E_{3}\left(P_{n} K_{(1, m))}\right.} x^{\left[\left(d_{u}\right)^{2}+\left(d_{v}\right)^{2}\right]}+\sum_{u v \in E_{4}\left(P_{n} K_{(1, m))}\right.} x^{\left[\left(d_{u}\right)^{2}+\left(d_{v}\right)^{2}\right]}+\sum_{u v \in E_{5}\left(P_{n} K_{(1, m))}\right.} x^{\left[\left(d_{u}\right)^{2}+\left(d_{v}\right)^{2}\right]}+\sum_{u v \in E_{6}\left(P_{n} K_{(1, m))}\right.} x^{\left[\left(d_{u}\right)^{2}+\left(d_{v}\right)^{2}\right]} \\
=\left|E_{1}\left(P_{n} K_{(1, m)}\right)\right| x^{13}+\left|E_{2}\left(P_{n} K_{(1, m)}\right)\right| x^{18}+\left|E_{3}\left(P_{n} K_{(1, m)}\right)\right| x^{4+(m+1)^{2}}+\left|E_{4}\left(P_{n} K_{(1, m)}\right)\right| x^{9+(m+2)^{2}} \\
+\left|E_{5}\left(P_{n} K_{(1, m)}\right)\right| x^{(m+1)^{2}+(m+2)^{2}}+\left|E_{6}\left(P_{n} K_{(1, m)}\right)\right| x^{2(m+2)^{2}}
\end{gathered}
$$




$$
=2 m x^{13}+m(n-3) x^{18}+2 m x^{4+(m+1)^{2}}+m(n-2) x^{9+(m+2)^{2}}+2 x^{(m+1)^{2}+(m+2)^{2}}+(n-3) x^{2(m+2)^{2}} .
$$

Theorem 3.9. The forgotten index or F-index of $P_{n} K_{(1, m)}$ is:

$$
\begin{gathered}
F\left(P_{n} K_{(1, m)}\right)=26 m+18 m(n-2)+2 m\left(4+(m+1)^{2}\right) \\
+m(n-2)\left(9+(m+2)^{2}\right)+2\left((m+1)^{2}+(m+2)^{2}\right)+2(n-3)(m+2)^{2} .
\end{gathered}
$$

Proof. By the definition of the forgotten index:

$$
\begin{aligned}
& F(G)=\sum_{u v \in E\left(P_{n} K_{(1, m)}\right)}\left[\left(d_{u}\right)^{2}+\left(d_{v}\right)^{2}\right]=\sum_{u v \in E_{1}\left(P_{n} K_{(1, m)}\right)}\left[\left(d_{u}\right)^{2}+\left(d_{v}\right)^{2}\right]+\sum_{u v \in E_{2}\left(P_{n} K_{(1, m)}\right)}\left[\left(d_{u}\right)^{2}+\left(d_{v}\right)^{2}\right] \\
& +\sum_{u v \in E_{3}\left(P_{n} K_{(1, m)}\right)}\left[\left(d_{u}\right)^{2}+\left(d_{v}\right)^{2}\right]+\sum_{u v \in E_{4}\left(P_{n} K_{(1, m)}\right)}\left[\left(d_{u}\right)^{2}+\left(d_{v}\right)^{2}\right] \\
& +\sum_{u v \in E_{5}\left(P_{n} K_{(1, m)}\right)}\left[\left(d_{u}\right)^{2}+\left(d_{v}\right)^{2}\right]+\sum_{u v \in E_{6}\left(P_{n} K_{(1, m)}\right)}\left[\left(d_{u}\right)^{2}+\left(d_{v}\right)^{2}\right] \\
& =13\left|E_{1}\left(P_{n} K_{(1, m)}\right)\right|+18\left|E_{2}\left(P_{n} K_{(1, m)}\right)\right| \\
& +\left|E_{3}\left(P_{n} K_{(1, m)}\right)\right|\left(4+(m+1)^{2}\right)+\left|E_{4}\left(P_{n} K_{(1, m)}\right)\right|\left(9+(m+2)^{2}\right) \\
& +\left|E_{4}\left(P_{n} K_{(1, m)}\right)\right|\left(9+(m+2)^{2}\right)+\left|E_{5}\left(P_{n} K_{(1, m)}\right)\right|\left((m+1)^{2}+(m+2)^{2}\right) \\
& +\left|E_{6}\left(P_{n} K_{(1, m)}\right)\right|\left((m+2)^{2}+(m+2)^{2}\right) \\
& =26 m+18 m(n-2)+2 m\left(4+(m+1)^{2}\right)+m(n-2)\left(9+(m+2)^{2}\right)+2\left((m+1)^{2}+(m+2)^{2}\right)+2(n-3)(m+2)^{2} .
\end{aligned}
$$

\section{Conclusion}

In this paper, many topological indices and polynomials were calculated. The most important which was the Mpolynomial, through which many indices were calculated through processes of integration and derivation of $\mathrm{M}$ polynomial, and we can calculated indices and polynomials for some special graph.

\section{References}

[1] H. Hosoya, On some counting polynomials in chemistry, Discrete Appl. Math. 19 (1988) 239-257.

[2] E. Deutsch, S. Klavžar, Computing Hosoya polynomials of graphs from primary subgraphs, MATCH Commun. Math. Comput. Chem. 70 (2013) 627-644.

[3] M. Eliasi, A. Iranmanesh, Hosoya polynomial of hierarchical product of graphs, MATCH Commun. Math. Comput. Chem. 69 (2013) 111-119.

[4] X. Lin, S. J. Xu, Y. N. Yeh, Hosoya polynomials of circumcoronene series, MATCH Commun. Math. Comput. Chem. 69 (2013) 755-763.

[5] I. Gutman, A formula for the Wiener number of trees and its extension to graphs containing cycles, Graph Theory Notes New York. 27 (1994) 9-15.

[6] H. Wiener, Structural determination of paraffin boiling points, J. Am. Chem. Soc. 69 (1947) 17-20.

[7] G. G. Cash, Relationship between the Hosoya polynomial and the hyper-Wiener index, Appl. Math. Lett. 15 (2002) 893-895.

[8] F. M. Brückler, T. Doŝlić, A. Graovac, I. Gutman, On a class of distance-based molecular structure descriptors, Chem. Phys. Lett. 503 (2011) 336-338.

[9] E. Deutsch, S. Klavžar. M-Polynomial, and degree-based topological indices. Iran. J. Math. Chem. 6 (2015) 93-102.

[10] M. Munir, W. Nazeer, S. Rafique and S. Kang, M. Mpolynomial and related topological indices of Nanostar $\begin{array}{lllll}\text { dendrimers. Symmetry. } 8 & \text { (2016) } 97 . & \text { doi: }\end{array}$ 10.3390/sym8090097.

[11] M. Munir, W. Nazeer, Z. Shahzadi and S. M. Kang, Some invariants of circulant graphs. Symmetry 8 (2016) 134. doi: $10.3390 /$ sym 8110134 .

[12] I. Gutman and N. Trinajstic, Graph theory and molecular orbitals total $\varphi$-electron energy of alternant hydrocarbons, Chem. Phys. Lett. 17 (1972) 535-538.

[13] K. Das and I. Gutman, Some properties of the second Zagreb index, MATCH Commun. Math. Comput. Chem. 52 (2004) $103-112$.

[14] I. Gutman and K. C. Das, The first Zagreb indices 30 years after, MATCH Commun. Math. Comput. Chem. 50 (2004) 83-92.

[15] S. Nikolić, G. Kovačević, A. Miličević and N. Trinajstić, The Zagreb indices 30 years after, Croat. Chem. Acta. 76 (2003) $113-124$.

[16] N. Trinajstic, S. Nikolic, A. Milicevic and I. Gutman, On Zagreb indices, Kem. Ind. 59 (2010) 577-589.

[17] D. Vukičević and A. Graovac, Valence connectivity versus Randić, Zagreb and modified Zagreb index: A linear algorithm to check discriminative properties of indices in acyclic molecular graphs, Croat. Chem. Acta. 77 (2004) 501-508.

[18] A. Milicevic, S. Nikolic and N. Trinajstic, On reformulated Zagreb indices, Mol. Divers. 8 (2004) 393-399. 
[19] M. Randić, On the characterization of molecular branching, J. Amer. Chem. Soc. 97 (1975) 6609-6615.

[20] B. Bollobas and P. Erdös, Graphs of extremal weights, Ars Combin. 50 (1998) 225-233.

[21] D. Amic, D. Beslo, B. Lucic, S. Nikolic and N. Trinajstić, The vertex-connectivity index revisited, J. Chem. Inf. Comput. Sci. 38 (1998) 819-822.

[22] Y. Hu, X. Li, Y. Shi, T. Xu and I. Gutman, On molecular graphs with smallest and greatest zeroth- Corder general Randić index, MATCH Commun. Math. Comput. Chem. 54 (2005) 425-434.

[23] B. Furtula, A. Graovac and D. Vukičević, Augmented Zagreb index, J. Math. Chem. 48 (2010) 370-380.

[24] Y. Huang, B. Liu and L. Gan. Augmented Zagreb Index of Connected Graphs. MATCH Commun. Math. Comput. Chem. 67 (2012) 483-494.

[25] V. K. Gupta, V. Lokesha, S. B. Shwetha and P. S. Ranjini, On the symmetric division deg index of graph, Southeast Asian Bull. Math. 40 (2016) 59-80

[26] A. T. Balaban, Highly discriminating distance based numerical descriptor, Chem. Phys. Lett. 89 (1982) 399-404.

[27] S. Fajtlowicz, On conjectures of Graffiti II, Congr. Numer. 60 (1987) 189-197.

[28] O. Favaron, M. Mahéo and J. F. Saclé, Some eigenvalue properties in graphs (conjectures of Graffiti-II), Discrete Math. 111 (1993) 197-220.

[29] G. H. Shirdel, H. R. Pour and A. M. Sayadi, The hyper-Zagreb index of graph operations. Iran. J. Math. Chem. 4 (2013) 213 220 .

[30] M. Ghorbani and N. Azimi, Note on multiple Zagreb indices. Iran. J. Math. Chem. 3 (2012) 137-143.

[31] R. Shaheen, S. Mahfud and Q. Alhawat, Some topological indices and polynomials of Cartesian Product of two Paths. Submitted to Bulletin of Computational Mathematics (2019). 